\title{
Information technology integration processes for the sustainable development of construction
}

\author{
Pirko Dmitry, Mayilyan Lia, Triputa Ivan, and Zelentsov Leonid* \\ Don State Technical University, Rostov-on-Don, 344000, Russian Federation
}

\begin{abstract}
The paper discusses the issues of creating a single information space for all participants in the investment and construction project, from the investor to the equipment manufacturers. The analysis of the existing information technologies used in the ICP management made it possible to distinguish four main classification groups. Of particular interest are the so-called integrator systems focused on combining the solution of tasks of certain functional areas of ICP management on one digital platform. Integrator systems, with all their merits, are a set of local information technologies and specialized software performing the functions of exchanging information between them using agreed protocols. At the same time, the main issue that was and remains on the agenda in the field of digitalization of the ICP management system is not being resolved - the creation of an information base that suits both designers and builders in terms of completeness and detail. At the Don State Technical University (DSTU), work is underway to create an intelligent ICP management system, which will ensure the integration of all stages of ICP management by creating an information base at the design stage in the BIM process that allows solving all organizational and technological problems both at the stages of COP, WPP and during operational management of the erection of construction facilities.
\end{abstract}

\section{Introduction}

The digitalization of construction based on the BIM (Building Information Modeling) system involves the automation of all stages and procedures throughout the entire life cycle of a facility. BIM technology is widespread in the West, where not only 3D modeling is already practiced, but also 5D, 6D and even 7D, and these technologies are used both at the design and construction stages [1].

\section{Materials and Methods}

If we consider the practice of European countries and the United States, it can be noted that they have been developing the concept of integrated construction for a long time. This implies the presence of a single information space based on the WEB for all project participants, from the investor to the equipment manufacturers. For our country, this

\footnotetext{
*Corresponding author:1.zelencov@yandex.ru
} 
problem is even more urgent than abroad, since in the existing management system for investment and construction projects (ICP), each control loop has its own local information technology (IT) that does not have the necessary information interaction with related technologies [2].

Information interaction of the design and construction stages is usually carried out through the transfer of information, both on paper and in the form of electronic files with different digital formats. As a result, there is a discrepancy not only in digital formats, but also in the degree of detail of the information transmitted from the design stage, which, in turn, does not allow creating a database that would satisfy the processes of construction production management quickly and with high accuracy.

Construction activity is characterized by the uniqueness of the manufactured products, its high material consumption, labor intensity and a number of other characteristics. Therefore, the existing "universal" control systems cannot fully cover all functional areas of management of design and construction activities. The efficiency of ICP management directly depends on the way of organizing the information environment, on-line transmission and provision of the required information.

Nowadays, there are information systems and technologies of various classes used in the construction industry:

- ERP systems;

- information technology of BIM;

- information technologies for the automation of the activities of design, construction companies and enterprises of the construction industry;

- integrators systems focused on combining technologies covering certain functional areas of ICP management on one digital platform.

In the software market, ERP systems are represented by the following companies: Galaktika, PARUS, 1C, KOMPAS, etc. [3,4,5].

The main disadvantage of such systems is the lack of mechanisms of integration into the production management system of design and construction companies.

In the design, the area of creating specialized information technologies was developed, the concept of which is based on the BIM methodology [1]. Revit [6, 7], Renga Structure [8] are the most popular technologies in the field of BIM in Russia.

The design algorithm using these technologies is the following sequence of actions:

- preliminary "decomposition" of the future building in height and horizontal is performed, which allows creating a "skeleton" of the future model;

- this "skeleton" is filled with elements from a previously created database of normative and reference information ("families" in Revit terminology). This approach to design allows speeding it up and reducing the number of errors that occur when manually entering information;

- additional elements, such as "Rooms" and "Zones", are placed and configured in the "skeleton" of the model;

- the building is analyzed for compliance with architectural, structural, sanitary, fire safety and other requirements;

- $\quad$ creation of "fragments" of the model - floor plans, facades and sections, etc.;

- drawings are supplemented with annotations: leaders, text notes, tabular data.

- drawings are being printed or the 3D model and documentation are being transferred, for example, for later use in other software.

BIM technologies for accelerating the work of estimation engineersand planners are implemented using a combination of Autodesk Revit and Estimo.Connect [9]

The Estimo.Connect interface allows the estimator not only to form and customize the data table, but also to get a complete picture of the project due to the presence of a $3 \mathrm{D}$ model. Table cells correspond to model elements, which allows working with them both 
from the model and from the table. This implementation of the estimate program allows the specialist to focus on optimizing the scope of work, to understand more deeply how the construction process will be organized, and only after that select the required reference book of norms and prices, determine the list of works for developing the estimate and generate cost estimates. The result obtained is exported to any estimate complex (for example, Grand Smeta, Smeta.RU, Gosstroismeta 2.0, etc.), finalized according to all standards, and drawn up as a standard estimate.

Renga Structure is the first domestic BIM system for designers and design engineers for creating an information model of a building or structure. This development has less functionality compared to Revit, but in recent years it has become a very competitive product. The developer of Renga is the Ascon company, which is simultaneously developing another information system Pilot-ICE, which is a corporate management system for a project organization and information modeling processes [8]. Both of these systems are developed on the same digital platform and together have the functionality to solve a wide range of problems in the field of design and project management.

Nowadays, systems have appeared on the software market that are focused on integrating technologies covering certain functional areas of investment and construction project management on one digital platform. Such integrator systems include: Lement Pro Building1, ADEPT, Assistant Build.

The most prominent representative of this approach is Lement Pro Building (developed by Element LLC). The Lement Pro Building system provides end-to-end management of information about a capital construction object using a BIM model at all stages of its life cycle: design, construction, operation. The system makes it possible to manage every element of the building, which is associated with the processes, tasks, documents, goals of the company.

The result of the implementation of a digital construction system using Lement Pro Building is the transparency of the organizational and technological processes of construction online, a significant reduction in communication costs, risks of information loss, the time for approval of construction documentation and changes in the construction process, the creation of an information system for operation during construction:

- at the design stage: the main goal is to increase the speed and quality of design;

- at the construction stage - to make an automated conveyor with electronic issuance of daily, weekly tasks and with automatic recording and control of the work performed;

- at the operation stage - to provide an operation information system with a virtual analogue of the building, where one can get all the technical documentation for any element, history (who installed it and when, when it needs to be serviced).

Main features of the ADEPT system:

- Unification of subdivisions in a single information field. Exchange of information and automation of work of employees of different departments within the framework of construction projects

- Work schedules based on accurately loaded estimates

- $\quad$ Automatic unloading of information from estimates. It can be changed to actually used one.

- $\quad$ Parallel analysis of estimated, planned and actual data

- $\quad$ Resource management - materials, mechanisms, people

- $\quad$ Planning the supply of materials, movement of machines and workers

- $\quad$ BIM during the construction phase: from $3 \mathrm{~d}$ model to $3 \mathrm{~d}$ fact of completion

- Automation of the chain: 3d model - estimate - work schedule - executive documentation - $3 \mathrm{~d}$ fact of completion in Navisworks [8].

In the process of managing the ICP, the system allows solving the following tasks.

For customers: 
planning of construction works, control of work performance and money consumption, visual control of the $3 \mathrm{~d}$ fact of completion based on data unloaded from $3 \mathrm{~d}$;

General contractors:

planning the work schedule and project budget, exchange of information between departments, plan-fact analysis.

For contractors:

planning of work execution and supply of resources, warehouse accounting, project budgeting, control of work execution.

The Assistant Build information complex [9] allows controlling the allocated budget and construction time (from obtaining a construction permit to putting the facility into operation), combining data on the facility into a single system, which supports the BIM ideology of information availability at any stage and at any moment of the object's life cycle (Figure 1).

The developed Assistant Build software has the following capabilities:

- synchronization of the delivery time of material resources with labor resources and construction equipment;

- monitoring the performance of work and compliance with the deadlines for the production of work according to the construction schedule;

- analysis of planned and actual indicators of construction;

- formation of assignments for subcontractors;

- preparation of analytical reports on the progress of construction;

- maintaining an archive of design and as-built documentation and documentation on the use of materials for the entire period of construction of the facility;

- uploading of local, object estimates and summary estimate calculation into the system;

- determining the cost and labor costs in accordance with the work schedule:

- visualization in Navisworks of individual materials and equipment, groups of objects, building blocks, construction progress by combining graphics and 3D models [9];

- creating and updating "as designed" and "as built"information models in Navisworks.

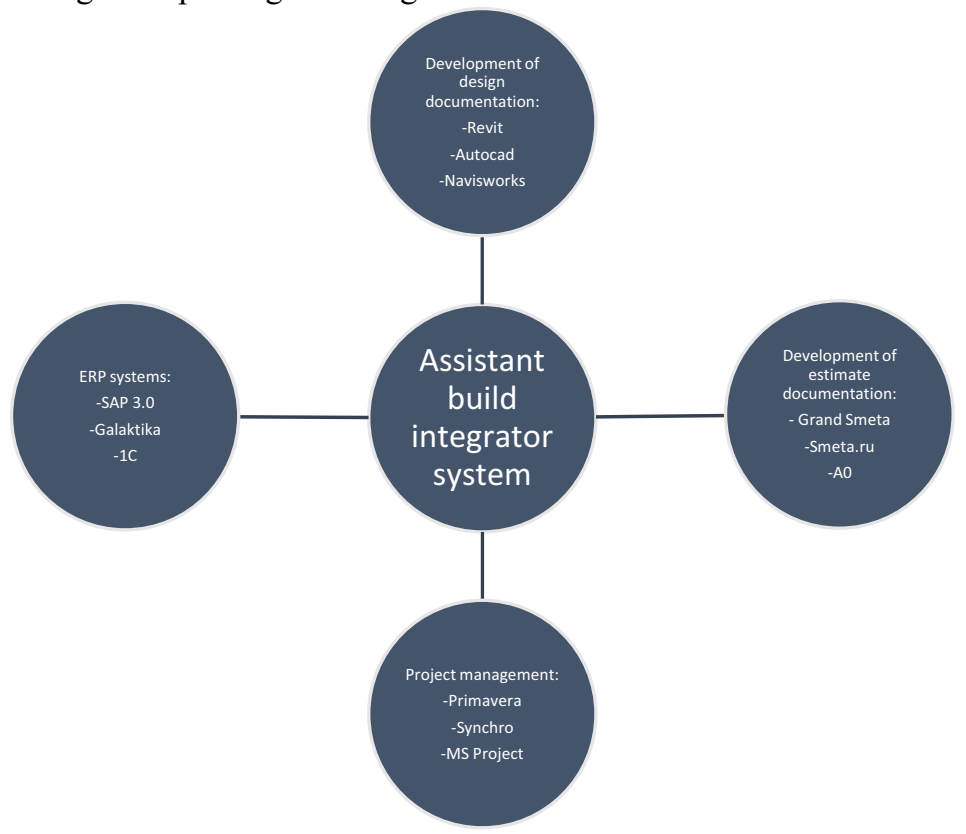

Fig.1. Structure of the Assistant Build integrator system 


\section{Results and Discussion}

Integrator systems, with all their merits, are a set of local information technologies and specialized software performing the functions of exchanging information between them using agreed protocols. This leads to the fact that it is very difficult to work in such a system without the help of a highly qualified consultant, since each application has its own database with an individual interface.

In addition, from our point of view, the disadvantages of existing integrator systems include the absence of:

- a unified information base, which does not allow one to fully use the advantages of BIM in the process of managing the life cycle of material resources and is the main limitation in their widespread use [1];

- a subsystem of operational management, which would ensure the daily recording, transmission and accumulation of reliable information about the course of production processes at the construction site;

- an autonomous management accounting system that allows carrying out a plan - factor analysis of the use of resources in kind and in value term synchronously with the production process;

- forecasting systems for time and cost parameters of a construction project.

The main issue that has been and remains on the agenda in the field of digitalization of the ICP management system is the creation of an information base that suits both designers and builders in terms of completeness and detail.

At the Don State Technical University (DSTU), work is underway to create an intelligent management system for investment and construction projects (IMS ICP) (Figure 2), which includes intelligent management systems for: design (IMS "Design"), construction (IMS "Construction"), operation (IMS "Operation"), as well as the information system used in the customer-developer-contractor control loop (IS “Customer-developer”) [3].

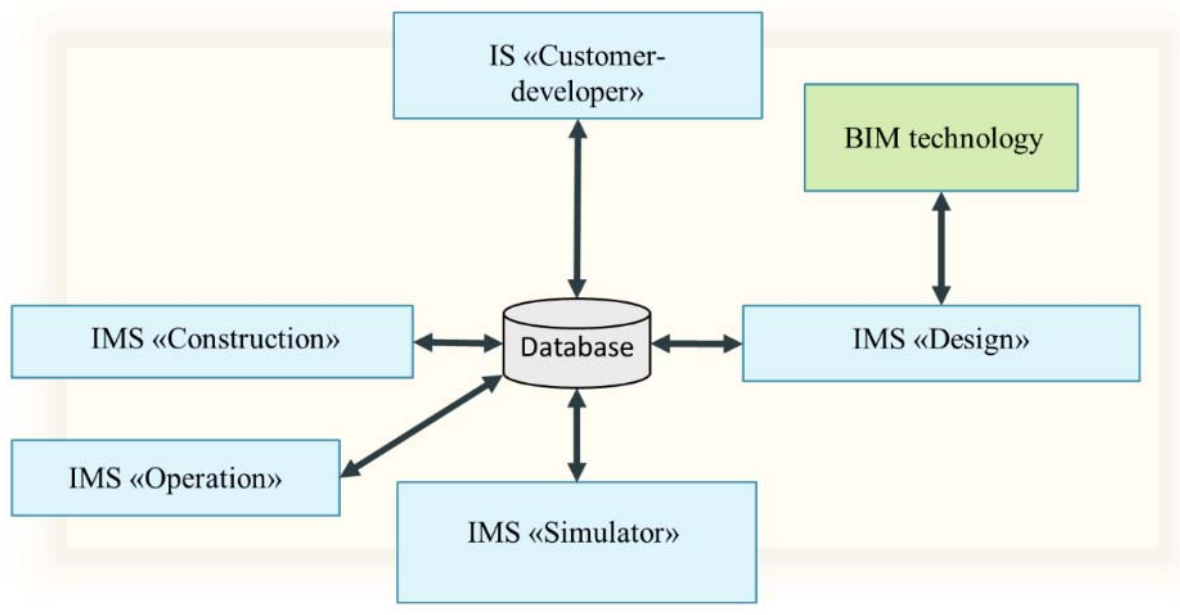

Fig. 2. Schematic diagram of the interaction of information technologies integrated into the IMS ICP

\section{Conclusions}

The main task of creating an integrated hierarchically organized information technology is to ensurethe use of detailed information at each subsequent level of ICP management, which arises at the previous level and takes into account the peculiarities of regulation of 
construction in the Russian Federation. The integration of all these systems is carried out by creating an information base at the design stage in the BIM process, which allows solving all organizational and technological problems, both at the stages of COP, WPP and during operational management of the erection of construction facilities.

\section{References}

1. L.B.Zelentsov et al., Construction production 2, 29-34 (2020)

2. A.A. Lapidus, A.O. Feldman, Scientific Review 21, 313-316 (2015)

3. L.B. Zelentsov et al., Construction production 1, 41-44 (2020)

4. L.B. Zelentsov et al., Intelligent control systems in construction (DSTU, 2017)

5. S.A. Nechaev, Days of student science: proceedings of scientific and technical conference (Publishing house of MISI-MGSU, Moscow, 2019)

6. O. Salosin et al., IOP Publishing 1614, 012028 (2020) doi:10.1088/17426596/1614/1/012028

7. E. Kochurina, E3S Web of Conferences 110, 01082 (2019)

8. S. Shulzhenkoet al., E3S Web of Conferences 164, 10031 (2020)

9. D.V.Topchiy et al., International Journal of Civil Engineering and Technology 10(2), 2160-2166 (2019) 